\title{
Features of a Sample of Star-forming Galaxies in two Adjacent SBS Fields
}

\author{
S.A. Hakopian * \\ V.Ambartsumian Byurakan Astrophysical Observatory (BAO), Armenia
}

\begin{abstract}
Some results are presented on studies of a sample of about hundred galaxies ("100SBS") from two adjacent fields of SBS (Second Byurakan Survey), which have spectral data in SDSS (Sloan Digital Sky Survey-www.sdss.org ). The work is carried out as a part of the long-term program (Hakopian, 2013) for in-depth study of the galaxies in seven selected SBS fields (including the two under investigation).

Comparative analysis was done between two classifications that the 100SBS objects got - from one side Starburst or Starforming in SDSS, from the other - SfGcont and SfGneb, with a possibility of further detailing in accordance to our scheme for SfG, i.e. star-forming galaxies. Obtained results showed good perspectives in using of our scheme for differentiation of activity phases of SfGs and for better statistics and comprehensive studies. Space distribution of 100 SBS galaxies,what is important, homogeneously selected in continuous (32 sq.deg) area, was obtained using uniformly determined in SDSS redshift values.As it follows from the graphs plotted at 0.01 intervals the maximum of the distribution, with an equal number of Staforming and Starburst galaxies falls on the range $0.01<\mathrm{z}<0.019$. The minimum of the distribution falls on the range $0.02<z<0.029$ and more expressed for Starbursts. Also some difference is discerned in a diversity of morphological appearance of the galaxies composing two neighboring to it intervals.
\end{abstract}

Keywords: SBS survey : Star-forming galaxies: Classificatons: Space ditribution.

\section{Introduction}

The Second Byurakan Survey (SBS) (Markarian \& Stepanian, 1983) was undertaken as a continuation of the Markarian/First Byurakan Survey (FBS) (Markarian et al., 1977) in a searching of active galaxies. Table 1 provides basic information on the survey conduction and the objects selection. Improved methods allowed a fainter limit on photographic plates to be reached, so more objects were detected, in particular, galaxies with emission lines. Most of them are currently being studied as galaxies of star-formation activity, which due to spatial distribution can be considered carriers of the main characteristics of the nearby Universe.

Comprehensive study of star-forming galaxies is an important part of our long-term program. The key role in it is played by SBS galaxies in separate fields, as namely these subsamples can be considered the most homogeneous in the survey. Seven fields were selected (see Fig. 1) out of 65 due to the higher level of completeness by the $\mathrm{V} / \mathrm{V}_{\max }$ test and other details. In the first phase of the program, the follow-up spectroscopy of all approximately 500 objects, constituting seven subsamples, was completed and their basic data, such as redshift values and type of activity, were obtained in accordance with a classification scheme adapted to the spectral material. About $80 \%$ of the objects got classification SfG - star-forming galaxy.

To designate the fields, we use a serial number from 1 to 7 , based on the order in which the RA values of their centers increase. In a focus of this work are the samples of the 4th and 5th selected fields, which are highlighted in Fig. 1 by a red circle.

\section{SfG sample in two adjacent fields of SBS}

The 4 th and 5 th selected fields with centers at $11 \mathrm{~h} 30 \mathrm{~m}+59 \mathrm{deg}$ and $12 \mathrm{~h} 0 \mathrm{~m}+59 \mathrm{deg}$, respectively, are located side by side and cover a continuous area of the celestial sphere of approximately 32 square degrees. Classification SfG, i.e. star-forming galaxy got 116 out of 158 in all in the fields and approximately a hundred

*susannahakopian@yahoo.com 
Table 1. The Second Byurakan Survey

OBSERVATIONS

Byurakan 1-m Schmidt telescope

Low-dispersion prisms (deg): 1.5, 3,4

Photographic plates: Kodak IIIa-(J, J, F)

Two samples of candidates for active galaxies:

(hypersensibilization)

Filters: no, GG495, RG2

starlike morphology (s).

Criteria:

Dispersion $(\AA \mathrm{mm}): 1800(\mathrm{H} \gamma), 850(\mathrm{H} \gamma), 1100(\mathrm{H} \alpha)$

Spectral range(nm): 350-540, 490-540, 630-690

Fields: $65 \times 16$ sq.deg (1000 sq.deg area)

emission lines (e, e:),

uv-continuum $(1,2,3)$.
App.Mag limit (pg): $18.5-19.5$

SBS Selected fields

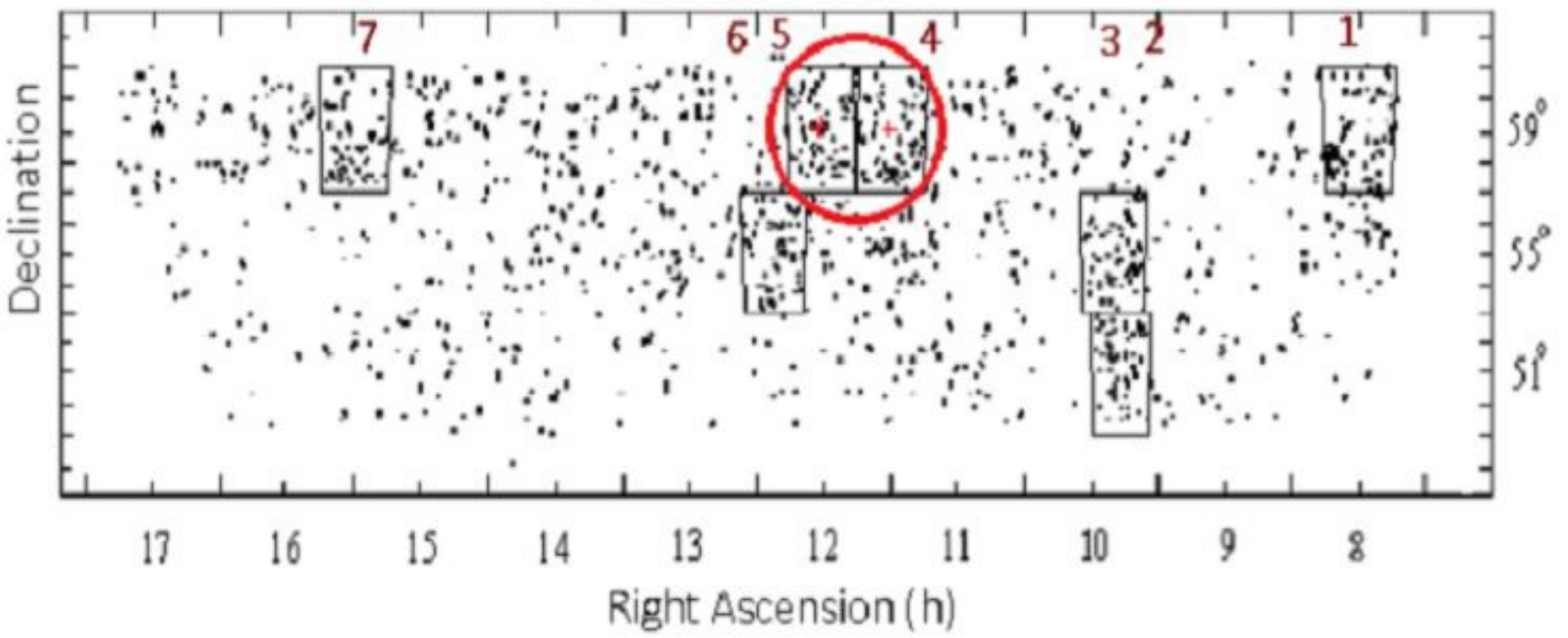

Figure 1. SBS galaxies and seven selected fields.

of 116 (further 100 SBS) are registered in SDSS (DR 16) with one at least associated spectrum (tied to the SBS designations in NED and/or LEDA). The uniformly obtained data of 100 SBS we use in comprehensive analysis. Some preliminary results, which obtained after comparative studies in spectral classifications and to get precise space distribution of the galaxies are given below.

\subsection{Spectral classifications}

We use the abbreviation SfG for star-forming galaxies to reference specifically to our classification scheme. In general, there is no unified approach to the use of the existing designations indicating the star formation of galaxies. This, in particular, makes it difficult to use them for statistical research or affects the results. Through our studies of homogeneous samples of such objects, we hope to make headway in improving classification schemes.

In our working classification scheme SfGs are divided on two types - SfGcont, i.e. star-forming in continual phase and SfGneb, i.e. star-forming in nebular phase (in some analogy with (Terlevich, 1997). Five numbered subclasses, from 1 to 5, are envisaged for each type in depend of available spectral detailing. The classification is based on the ascending of values of intensity and equivalent width of Balmer $\mathrm{H} \alpha$ line, begining from $\mathrm{EW}(\mathrm{H} \alpha)=5 \AA$ for SfGcont_1 and $\mathrm{EW}(\mathrm{H} \alpha)=100 \AA$ for SfGneb_1, taking into account the degree of manifestation of absorption lines.

Activity types that assigned in SDSS to 100 SBS galaxies in accordance to their spectra and imply activity of processes are two - Starforming or Starburst. In our scheme we also have two types (see above), but for each of them more detailing presupposed and the results we obtain in comparison show good effectiveness for spectral classification. Data on 5 chosen as examples galaxies are used to illustrate in short how SfG classification works in use.

Figure 2 shows spectra lined up from left to right in the order of increasing activity in correspondence 
to the SfG classifications, given below each.The image of the galaxy, each spectrum is associated, is shown directly below, in the upper row of images. The 15 galaxies imaged in three rows of Fig. 2 are all those from 100SBS which have $z<0.09$. The colour of frames of all SDSS images used through the paper indicates, by implication, SDSS activity type - brown means Starforming galaxy, blue - Starburst. A certain tendency can be traced visually along the sequence of spectra, at the same time considerable differences are seen both between spectra of two Starforming galaxies, and between the spectra of three Starbursts. Two other spectra classified as SfGcont_1 and SfGneb_5, i.e. completing the row on both sides, perfectly complement this sequence. They are shown in Fig. 3 together with images of the galaxies, which redshift values lie in the interval $0.01<\mathrm{z}<0.019$.
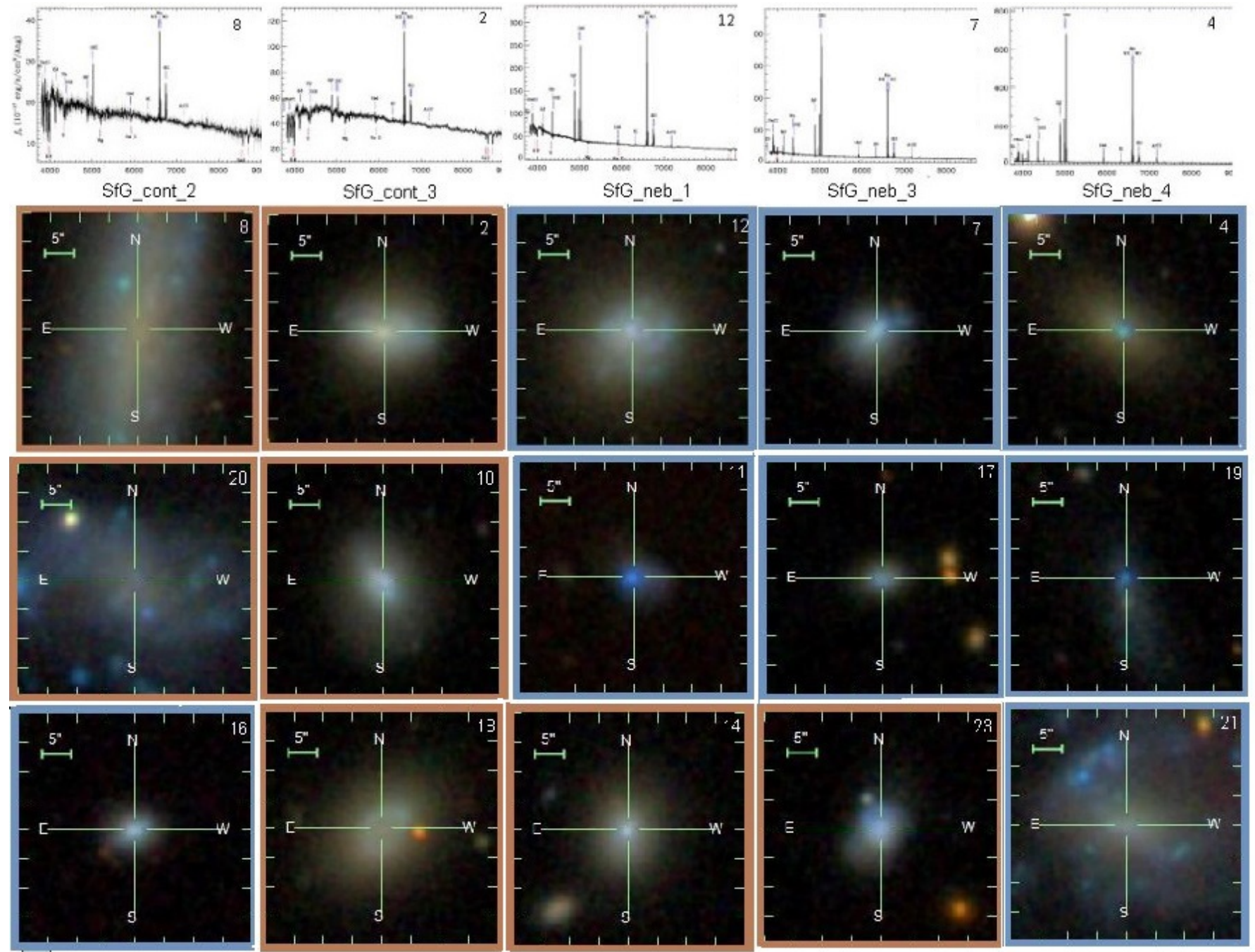

Figure 2. Some SDSS spectra and images of all 15 galaxies from 100 SBS with $\mathrm{z}<0.01$.
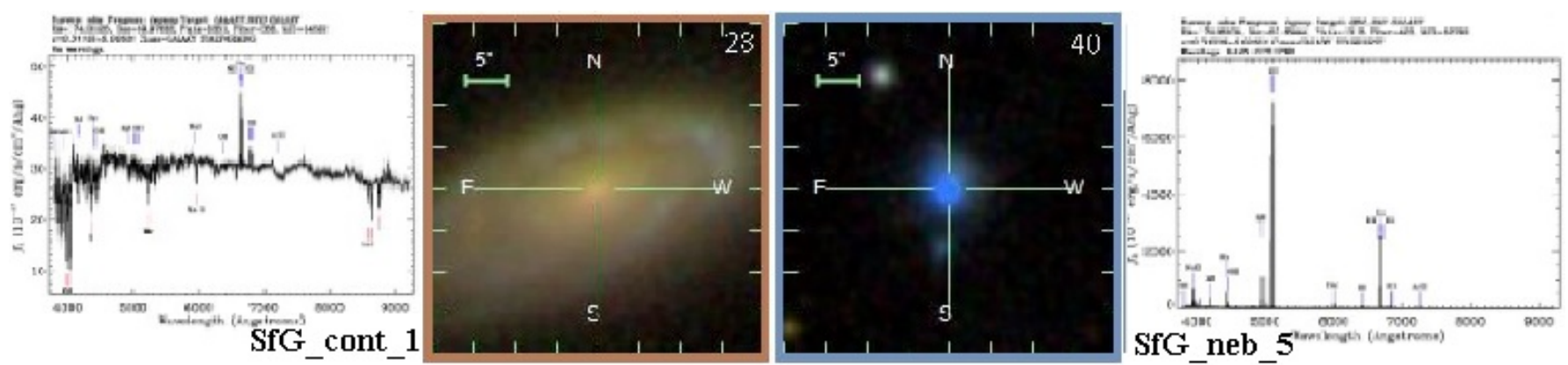

Figure 3. SDSS spectra and images of 2 galaxies from 100 SBS with $0.01<\mathrm{z}<0.019$.

Thus, it can be assumed that SfG classification has good perspectives. SDSS data on 100 SBS will be an excellent base to make significant improvements in the scheme concerning the quantitative parameters 
of the spectral lines and other features, including morphological structure of the galaxies.

Also, after the discussion on spectral classifications some clarifications are important.In the sample 100 SBS, which includes only those from SfG galaxies of the two fields classified Starforming or Starburst. Among 16, which remain out of it, 6 also have spectra in SDSS, but classified as Galaxy. Four of them are SfGcont_1, two - SfGcont_2 according to our scheme. It means, that a search in SDSS of starforming (in a wide meaning) galaxies (when completeness is important) by Starforming and Starburst types would be insufficient, and adding Galaxy type in search - meaningless. In this situation we see a manifestation of efficiency of the Second Byurakan Survey in its conduct and selection of objects.
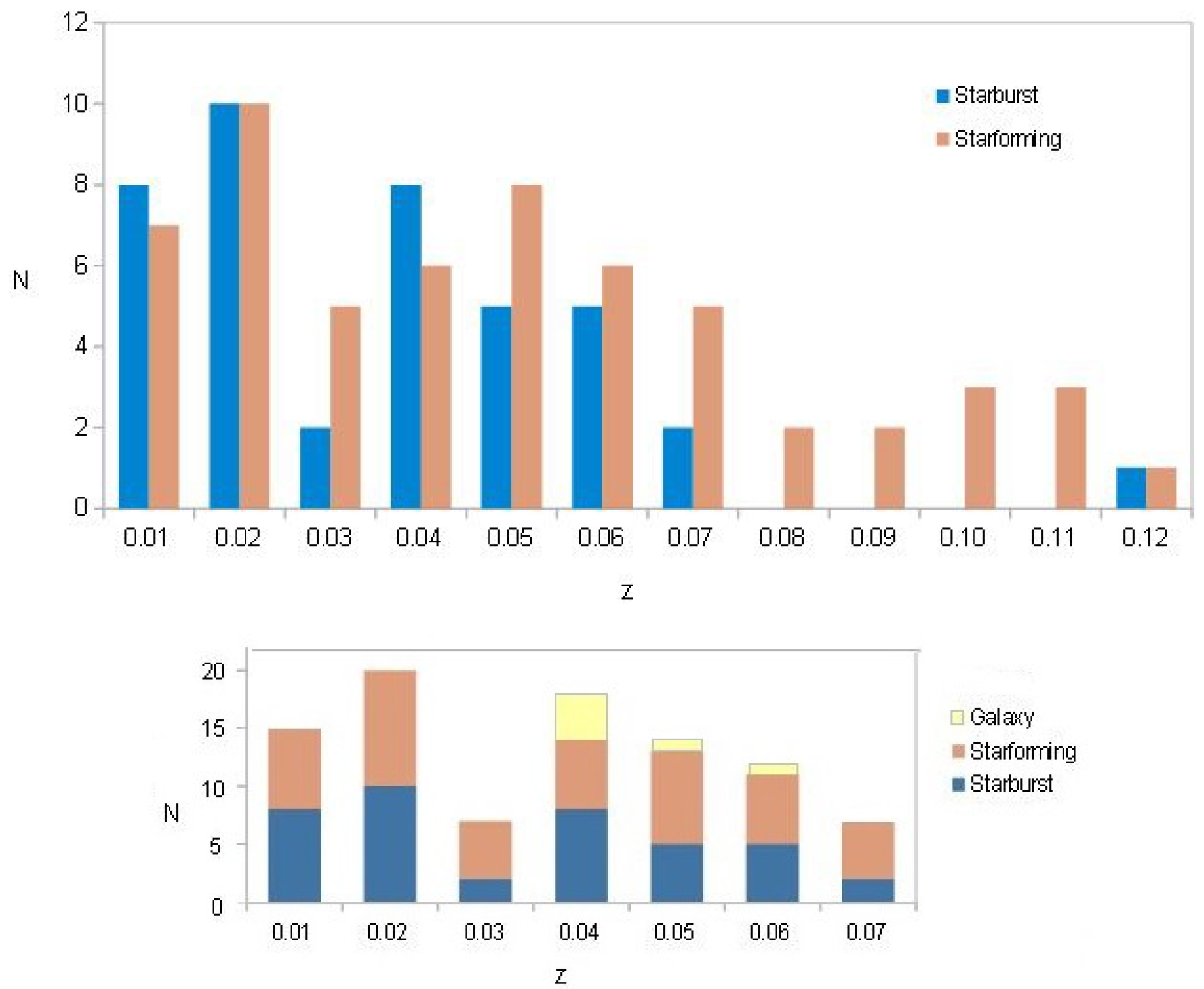

Figure 4. Two graphs of z-distribution of 100 SBS galaxies according to SDSS data

\subsection{Space distribution of $100 \mathrm{SBS}$ galaxies}

We used the uniformly determined SDSS redshift values to get as precise as possible space distribution of the 100SBS galaxies. By two columns of the upper histogram of Fig.4 the distribution illustrated separately for Starforming and Starburst galaxies up to $\mathrm{z}=0.12$ with an interval of 0.01. It should be borne in mind that the level of redshift completeness is achieved approximately at 0.07 . It depends on the details of the survey realization, such as spectral ranges of the used photographic plates, the registered emission lines and other (see Table 1 ).

Smaller number of Starbursts in comparison with Starformings at the values of $z>0.04$ is one of the features of the distribution, which is not clear, as usually Starburst are much brighter. So, that can be question on their space distribution. But the main feature in the range of reliability of the distribution, 
which is hard not to notice, - that the numbers of galaxies of both types in the range $0.02<\mathrm{z}<0.03$ is considerably less than in the neighboring intervals. And this minimum in the distribution looks deeper if we add $z$ - values of separately mentioned above objects that remain outside of 100 SBS because of SDSS type Galaxy. This is clearly seen in the diagram in the lower part of Fig. 4.
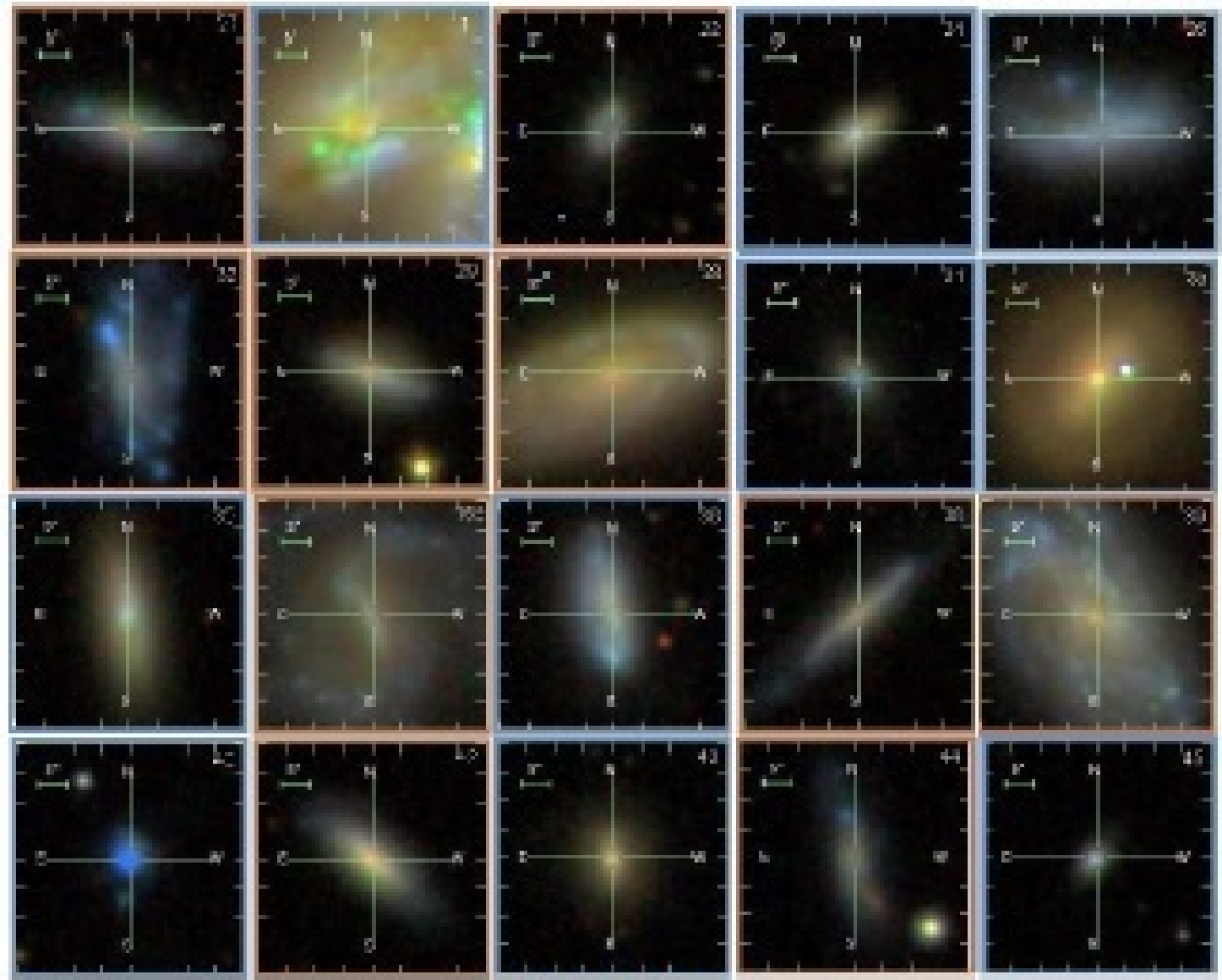

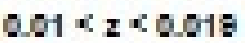

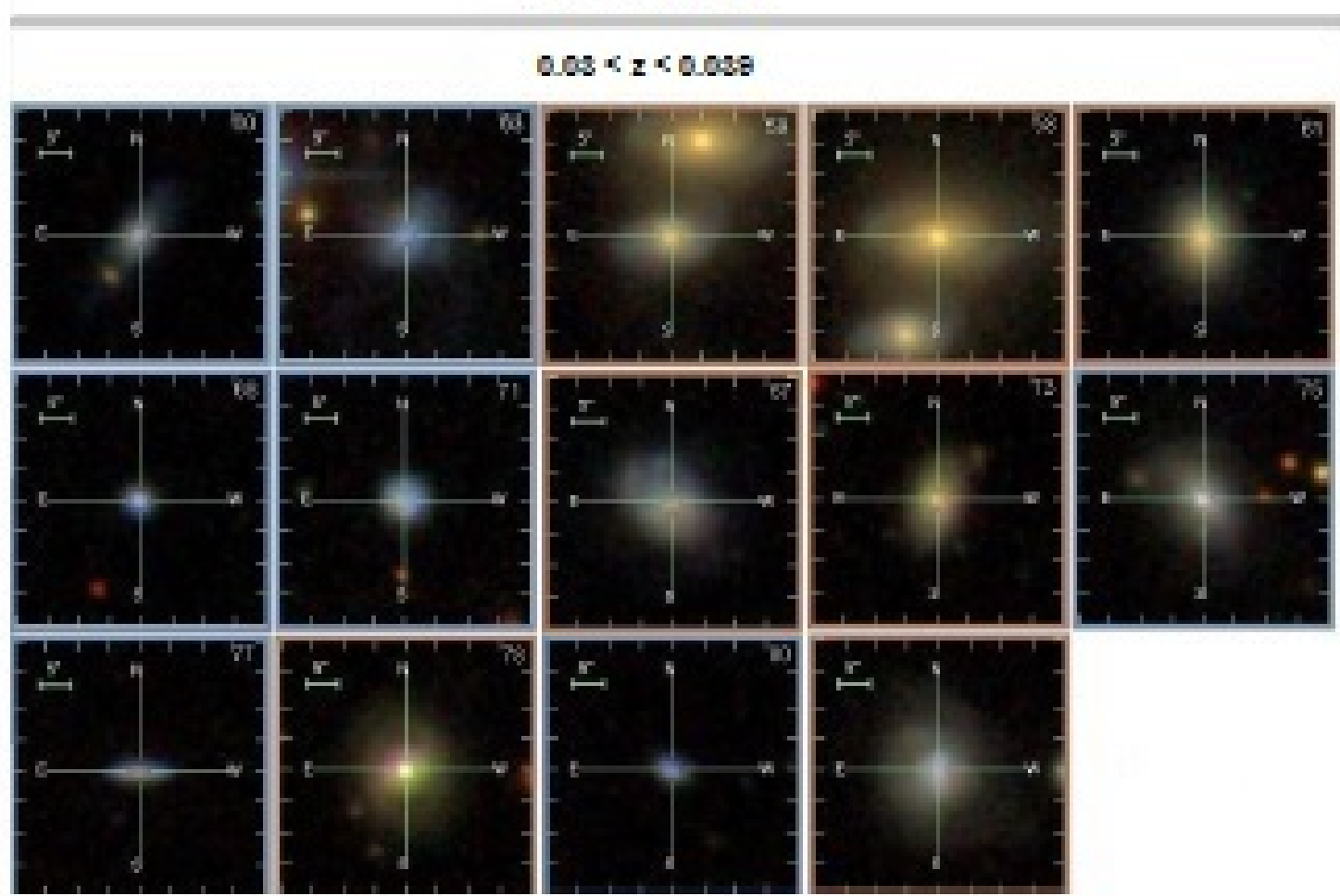

Figure 5. SDSS images of the galaxies from 100 SBS with $0.01<\mathrm{z}<0.019$ in the upper part, with $0.03<\mathrm{z}<0.039$ in the lower part of the figure. 
The peak in the redshifts distribution falls on the interval $0.01<\mathrm{z}<0.019$ with the same number of Starforming and Starburst galaxies, as it seen from the graphs of Fig. 4. The spectra of two of these objects were discussed above (see Fig. 3). Images of all 20 galaxies from this section are shown in the upper part of Fig.5, in the ascending order of $\mathrm{z}$ values - from left to right, row by row, starting from the top. Galaxies filling the interval $0.03<\mathrm{z}<0.039$ are shown in a similar sequence in the lower part of the Fig. 5. Attentive comparing of the images of galaxies in the upper part of Fig. 5 with the images in the lower part by a visual inspection allows us to make a preliminary assumption that there is some difference between appearance of the galaxies on either side of the interval with minimum of the $\mathrm{z}$ - distribution. On the left of it, among the closer objects, including the galaxies in Fig. 2, we see all the diversity of morphology and different spatial orientations. The galaxies on the other side of the minimum look pretty similar and this only applies to the interval $0.03<\mathrm{z}<0.039$. Among more distant galaxies again more diversity is observed - bright compact objects, developed structures, elongated shapes. Further studies will show how well this assumption is justified.

The dependence of the morphology and orientation of SfG galaxies on the spatial distribution is one of the questions of interest to us, directly related to the evolutionary chain of events.

\section{Conclusions}

Some results of a study of a sample of about 100 star-forming galaxies using SDSS data are presented.The sample (100 SBS) consists of SBS galaxies selected in two selected survey fields with common continuous area of 32 sq.deg. The list of 100 SBS designations and their data will be published subsequently.Comparing and juxtaposing of classifications that the galaxies got in SDSS with those in accordance to our scheme, which we name SfG classification, showed effectiveness and boundaries of the use of the latter. In the space distribution obtained with SDSS redshift values some peculiarities were revealed, which are required in further studies promising interesting results.More analysis on morphological and other features equally important for understanding of the formation and evolution of star-forming galaxies will be considered in following papers.

\section{Acknowledgements}

Funding for the Sloan Digital Sky Survey IV has been provided by the Alfred P. Sloan Foundation, the U.S. Department of Energy Office of Science, and the Participating Institutions. SDSS-IV acknowledges support and resources from the Center for High Performance Computing at the University of Utah. The SDSS website is www.sdss.org.

\section{References}

Hakopian S. A., 2013, arXiv:1403.0127,

Markarian B. E., Stepanian D. A., 1983, Astrofizika, 19, 14

Markarian B. E., Lipovetskii V. A., Stepanian D. A., 1977, Astrofizika, 13, 397

Terlevich R., 1997, in Franco J., Terlevich R., Serrano A., eds, Vol. 6, Revista Mexicana de Astronomia y Astrofisica Conference Series. p. 1 